OPEN ACCESS

Edited by:

Thorben Sprink,

Julius Kühn-Institut, Germany

Reviewed by:

Ghulam Kadir Ahmad Parveez, Malaysian Palm Oil Board, Malaysia Friedrich Fauser

Carnegie Institution for Science, USA

*Correspondence:

Hui Duan

hui.duan@simplot.com

Specialty section:

This article was submitted to

Plant Biotechnology,

a section of the journal

Frontiers in Plant Science

Received: 08 August 2016 Accepted: 05 October 2016

Published: 25 October 2016

Citation:

Forsyth A, Weeks T, Richael C and

Duan $H$ (2016) Transcription

Activator-Like Effector Nucleases

(TALEN)-Mediated Targeted

DNA Insertion in Potato Plants.

Front. Plant Sci. 7:1572.

doi: 10.3389/fpls.2016.01572

\section{Transcription Activator-Like Effector Nucleases (TALEN)-Mediated Targeted DNA Insertion in Potato Plants}

\author{
Adrienne Forsyth, Troy Weeks, Craig Richael and Hui Duan* \\ Simplot Plant Sciences, J.R. Simplot Company, Boise, ID, USA
}

Targeted DNA integration into known locations in the genome has potential advantages over the random insertional events typically achieved using conventional means of genetic modification. Specifically integrated transgenes are guaranteed to co-segregate, and expression level is more predictable, which makes downstream characterization and line selection more manageable. Because the site of DNA integration is known, the steps to deregulation of transgenic crops may be simplified. Here we describe a method that combines transcription activator-like effector nuclease (TALEN)-mediated induction of double strand breaks (DSBs) and non-autonomous marker selection to insert a transgene into a pre-selected, transcriptionally active region in the potato genome. In our experiment, TALEN was designed to create a DSB in the genome sequence following an endogenous constitutive promoter. A cytokinin vector was utilized for TALENs expression and prevention of stable integration of the nucleases. The donor vector contained a gene of interest cassette and a promoter-less plant-derived herbicide resistant gene positioned near the T-DNA left border which was used to select desired transgenic events. Our results indicated that TALEN induced T-DNA integration occurred with high frequency and resulting events have consistent expression of the gene of interest. Interestingly, it was found that, in most lines integration took place through one sided homology directed repair despite the minimal homologous sequence at the right border. An efficient transient assay for TALEN activity verification is also described.

Keywords: TALEN, gene targeting, potato, double stand break, transgenic, homologous recombination

\section{INTRODUCTION}

Plants can be improved for cropping purposes by inserting specific DNA sequences into their genomes. The DNA sequences are comprised of sequences that, when expressed in plants, confer a new trait to plants. Various methods have been developed for plant transformation that offer no control over the site into which introduced DNA sequences eventually reside in the genome. Such random insertions present several problems. Insertion can occur either in a transcriptionally active or inert region that may affect the expression level of the transgene (Peach and Velten, 1991). A transgene or a portion of DNA sequence could be inserted within a native gene sequence and consequently abolish the function of that gene. It is also possible to unintentionally create a new open reading frame at the junction of the insert and native DNA, which when expressed 
could negatively affect the quality or agronomic characteristics of the transgenic plant. Thus, given the unpredictable aspects of random insertion through transformation, introduction of a new trait into a crop requires generating a large number of transgenic lines. Subsequent analyses are also complicated due to the differences between all independent transgenic lines. Especially for vegetatively propagated crops like potato, trait introgression or insert elimination through crossing is complicated. The broad introduction of transgenic traits into asexually propagated crops would require genetic engineering of many commercially important varieties. Given the large number of transgenic lines needed to be produced in each variety, it is very difficult to justify financially.

For many reasons, targeted transgene integration has long been sought after in plant genetic modification. Original efforts utilized large stretches of sequence with homology to host target DNA to induce homologous recombination (HR), but very low targeted integration frequencies were achieved (Kempin et al., 1997; Hanin and Paszkowski, 2003). Subsequently, positivenegative selection methods were used to increase the efficiency of HR-mediated targeted integration in rice and Arabidopsis (Wang et al., 2001; Terada et al., 2002, 2007; Lida and Terada, 2005). The efficiency of HR based knock-in is also enhanced by modifying or expressing the components in the HR pathway, such as helicase $\operatorname{RecQ}$, resolvase ruvC and Rec8 (Shalev et al., 1999; Reiss et al., 2000; Li et al., 2004). Another approach of targeted integration utilizes site-specific recombinase-mediated integration systems (Fukushige and Sauer, 1992; Albert et al., 1995; Golic et al., 1997; Day et al., 2000). In this approach, DNA is inserted into a previously introduced recombination site in the genome which serves as a target. This is especially valuable for gene stacking when multiple site-specific recombination systems are combined. The newly integrated molecule contains a second recombination site that will serve as the target for the next round of DNA integration (Ow, 2011; De Paepe et al., 2013). Another approach was based on the observation that induced double strand breaks (DSBs) could facilitate targeted T-DNA integration in the plant genome (Puchta et al., 1993; Chilton and Que, 2003; Tzfira et al., 2003). In these studies, a specific rare endonuclease site, such as I-SceI was first randomly introduced into the plant genome and subsequently used as target site to generate a DSB. However, generating a DSB in a specific desired target locus still remains a challenge.

Endonuclease-based genome editing enzymes such as, meganucleases (Epinat et al., 2003), Zinc finger nucleases (ZFN) (Porteus and Baltimore, 2003), Transcription activator-like effector nucleases (TALEN) (Bogdanove and Voytas, 2011; Li et al., 2011) and CRISPR-associated (Cas) endonucleases (Jinek et al., 2012; Mussolino and Cathomen, 2013; Xie and Yang, 2013; Bortesi and Fischer, 2015) provide tremendous possibilities for generating DSBs in a targeted DNA region. Once this DSB occurs, the plant DNA repair machinery may either repair the break through a non-homologous end joining (NHEJ) pathway, which is imprecise and creates mutations to achieve gene knock out, or through a HR pathway to achieve gene replacement or insertion (Symington and Gautier, 2011). Several reports have shown the usage of these nucleases for targeted DNA integration in various plant species. These include: ZFN: Wright et al. (2005), Cai et al. (2009), Shukla et al. (2009), Townsend et al. (2009), Ainley et al. (2013), and De Pater et al. (2013); Talen: Zhang et al. (2013); Meganucleases: Puchta et al. (1993), Chilton and Que (2003), D'Halluin et al. (2008), D'Halluin et al. (2013) and Cas9: Li et al. (2013), Shan et al. (2013), and Svitashev et al. (2015).

In the present study, we describe a two-plasmid, TALENbased strategy to induce a DSB in a preselected location of the potato genome and integrate a gene of interest cassette into this site. More specifically, the first plasmid contains a pair of TALENs designed to induce DSB in the intron of the $5^{\prime}$ UTR of a constitutively expressed Ubiquitin7 (Ubi7) gene. The plasmid was designed to express TALENs transiently. If stable integration of the TALEN expression cassettes occurs, it will be negatively selected out. Targeted insertion of T-DNA is achieved when a second donor plasmid comprised of a promoterless mutated herbicide resistant gene (non-autonomous marker) and a gene of interest cassette are co-transformed into the potato plant. Herbicide resistant lines will develop only if the T-DNA is inserted into the targeted region and the transformants confer herbicide resistance. Herbicide resistant transformants were analyzed and our results indicated a high efficiency of targeted T-DNA integration. Although the original strategy of integration was based on NHEJ mechanism, it was discovered that the minimal homologous sequence at the right border resulted in one-sided HR-mediated integration.

\section{MATERIALS AND METHODS}

\section{Potato Transformation}

Ranger Russet potato (Solanum tuberosum cv Ranger Russet) tissue culture stock plants were maintained in magenta boxes containing $40 \mathrm{ml}$ half-strength Murashige and Skoog (MS) medium (Murashige and Skoog, 1962) modified by replacing ferrous sulfate and disodium EDTA with $36.7 \mathrm{~g} / \mathrm{l}$ of ferric sodium EDTA, $30 \mathrm{~g} / \mathrm{l}$ sucrose and $2 \mathrm{~g} / \mathrm{L}$ gelzan. All plants were maintained in a growth chamber at $24^{\circ} \mathrm{C}$ under a $16 \mathrm{~h}$ photoperiod. Plants were inoculated with a mixture of Agrobacterium containing pSIM2170 and pSIM2370 constructs. The explants were transferred onto co-culture medium containing one-tenth MS modified basal medium with Gamborg's vitamins (Gamborg, 1966), $30 \mathrm{~g} / \mathrm{l}$ sucrose and $6 \mathrm{~g} / \mathrm{l}$ agar on filter paper, under low light conditions. Explants were transferred and remained on callus induction medium (CIM) containing MS modified basal medium with Gamborg's vitamins, $2.5 \mathrm{mg} / \mathrm{l}$ zeatin riboside, $0.1 \mathrm{mg} / \mathrm{l}$ napthaline acetic acid, $30 \mathrm{~g} / \mathrm{l}$ sucrose, $300 \mathrm{mg} / \mathrm{l}$ timentin, $1.2 \mathrm{ml} / \mathrm{l}$ plant preservative mixture (PPM) and $6 \mathrm{~g} / \mathrm{l}$ agar. After 2 weeks, explants were transferred to CIM containing $2.0 \mathrm{mg} / \mathrm{l}$ imazamox for selection and maintained for 4 weeks, transferring to fresh medium every 2 weeks. Explants were then transferred to shoot induction medium (SIM) containing MS modified basal medium with Gamborg's vitamins, $2.5 \mathrm{mg} / \mathrm{l}$ zeatin riboside, $0.3 \mathrm{mg} / \mathrm{l}$ giberellic acid, $30 \mathrm{~g} / \mathrm{l}$ sucrose, $2.0 \mathrm{mg} / \mathrm{l}$ imazamox, $300 \mathrm{mg} / \mathrm{l}$ timentin, $1.2 \mathrm{ml} / \mathrm{l} \mathrm{PPM}$ and $6 \mathrm{~g} / \mathrm{l}$ agar. Explants were cultured on SIM for 6 weeks and transferred every 2 weeks to fresh medium. Shoots were recovered for 2 weeks and then were 
rooted on one-half strength MS modified basal medium with Gamborg's vitamins containing $300 \mathrm{mg} / \mathrm{l}$ timentin, $1.2 \mathrm{ml} / \mathrm{l} \mathrm{PPM}$ and $0.5 \mathrm{mg} / \mathrm{l}$ imazamox.

\section{Construct Preparation}

Transcription activator-like effector nuclease backbone originated from Hax3 TALE from Brassicaceae pathogen $X$. campestris pv. Armoraciae strain, a member of the AvrBs3 family. Plant codon optimized Hax3 sequence (Mahfouz et al., 2011) was synthesized by Invitrogen (Thermo Scientific, Waltham, MA USA) as three fragments: $\mathrm{N}$ terminal with a SV40 nuclear localization sequence (NLS), repeat variable diresidue (RVD), and truncated C-terminal with a FokI cleavage domain (Kim et al., 1997). The N-terminal and C-terminal fragments were used universally for both expression cassettes. The RVD was specifically designed for each DNA target. For a pair of effector nucleases, two expression cassettes were constructed. The first cassette (forward) contained a figwort mosaic virus (FMV) promoter and octopine synthase (OCS) terminator. The second cassette (reverse) contained a $35 \mathrm{~S}$ promoter and Nos terminator. Each TALEN expression cassette was assembled in the pBluescript SK (-) cloning vector (Agilent Technologies, Santa Clara, CA, USA) and both forward and reverse TALEN cassettes were assembled in a binary vector using conventional restriction enzyme cloning techniques.

The target plasmid, pSIM2167, used for testing transient TALEN efficiency was constructed by in-frame fusion of a $60 \mathrm{bp}$ target DNA sequence, including forward and reverse TALE binding sequences and a spacer, to a GUS reporter gene driven by an FMV promoter in a binary vector. In the spacer region of the sequence, there is an in-frame stop codon, as well as an AluI enzyme site. The donor plasmid was constructed by placing a promoter-less mutated potato $A L S$ gene ( $m S t A L S$ ) at the right border side followed by the gene of interest cassette. In this proof of concept study, the donor plasmid pSIM2370 contains a plant antibiotic resistant gene (nptII) expression cassette.

\section{Transient Infiltration}

A transient infiltration assay in Nicotiana benthamiana leaves was used to test TALEN function and activity. Wild type $N$. benthamiana plants were grown for Agrobacterium infiltration experiments and maintained under standard greenhouse conditions with an ambient temperature of $22-25^{\circ} \mathrm{C}$ and $16 \mathrm{~h}$ light period. For assays, mature leaves of 4-6 weeks old plants were used.

Two binary vectors were utilized in this assay, pSIM2167 provided the target sequence for the TALEN and PSIM2170 contained the pair of TALENs. Both binary vectors were transformed into Agrobacterium strain AGL1and the resulted Agrobacterium were used for infiltration assays. N. benthamiana leaves were co-infiltrated with a mix of pSIM2167 + pSIM2170 Agrobacterium cultures, as well as either pSIM2167 or pSIM2170 alone as negative controls into two zones on opposite leaf halves. Prior to infiltration a suspension of Agrobacterium harboring the target and TALENs were suspended in a 1:1 ratio which were coinfiltrated at a combined OD600 of 0.4 or independently at an
OD600 of 0.3. A $1 \mathrm{ml}$ syringe was used to deliver Agrobacterium into the abaxial side of third and fourth leaves from top.

\section{Gus Staining}

Transient GUS assays were performed to visualize TALEN activity in the co-infiltrated leaves. Three days post inoculation, two leaf disks of approximately $0.9 \mathrm{~cm}$ were collected. The leaf disks were subsequently incubated in X-Gluc (5-bormo4choror3-indolyl-betd glucuronide) staining solution, briefly vacuum infiltrated, incubated at $37^{\circ} \mathrm{C}$ overnight, and destained in $70 \%$ EtoH over a 2 days period. The leaves were then visualized under the microscope. Experiments were performed twice with similar results.

\section{Sequence Analysis}

To detect modifications in the target sequence of the infiltrated plants, $5 \mathrm{~mm}$ leaf disks were excised from the infiltrated leaf regions 2-3 days post infiltration. Genomic DNA was extracted using a CTAB based method (Porebski et al., 1997). Approximately $100 \mathrm{ng}$ of DNA was digested with AluI enzyme. Amplification was conducted with $20 \mathrm{ng}$ of the digested DNA in a PCR reaction containing $0.5 \mathrm{mM}$ primers $\mathrm{HD} 184 \mathrm{~F}$ and HD184R, which spans 200 bp on each side of the targeted region. The resulted PCR fragment was subsequently digested with AluI enzyme for the enrichment of the mutated target sequence. Undigested fragment (loss of AluI site) was cloned and multiple colonies were sent for sequence analysis for each of the treatment groups.

\section{PCR Screening and Genotyping}

Insertion specific primer pair HD175F and HD198R was designed and used for PCR genotyping for TALEN-mediated targeted insertion (Supplementary Table S1). DNA was isolated from tissue culture leaf material using a CTAB method (Porebski et al., 1997). DNA underwent PCR amplification and products were cloned and sequenced.

\section{Southern Blot}

Non-radioactive digoxigenin genomic Southern blotting was used to detect targeted DNA insertion and copy number. Plants were grown in the $\mathrm{GH}$ under conditions previously described. Genomic DNA was isolated via standard CTAB method from $0.5 \mathrm{~g}$ of young leaf tissue (Porebski et al., 1997). Six microgram of genomic DNA was digested with HindIII enzyme. The digested DNA was run on a $0.8 \%$ agarose gel in TBE buffer overnight. The gels were denatured and transferred to Hybond$\mathrm{N}+$ membrane (GE Healthcare Life Science, Pittsburg PA USA). A $1.1 \mathrm{~kb}$ DIG labeled DNA probe for StALS was synthesized by PCR amplification with the primer pair HD230F and HD230R (Supplemental Table S1) according to the manufacturer's protocol (Roche Diagnostic, Indianapolis, IN, USA). The DNA probe was heat denatured and added to the Roche DIG easy Hyb buffer and rotated at $42^{\circ} \mathrm{C}$ overnight. Washing and developing was carried out via manufacturer's instructions (Roche Diagnostic, Indianapolis, IN, USA). After exposure, the film was developed using a Konica SRX-101A developing machine. 


\section{Northern Blot}

RNA from field tissue was isolated using Tri Reagent according to the manufacturer's protocol (Sigma-Aldrich, St. Louis, MO, USA). Fifteen microgram of RNA was run on a standard $1 \%$ agarose gel in MOPS. The gels were washed with 10xSSC and blotted to Hybond-N+ membrane (GE Healthcare Life Science, Pittsburg, PA, USA). An nptII DIG labeled probe was synthesized with primers $\mathrm{HD} 288 \mathrm{~F}$ and HD288R to produce a $0.5 \mathrm{~kb}$ probe according to the manufacturer's protocol (Roche Diagnostic, Indianapolis, IN, USA). The DNA probe was heat denatured and added to the Roche DIG easy Hyb buffer and rotated at $42^{\circ} \mathrm{C}$ overnight. Washing and developing was carried out via manufacturer's instructions (Roche Diagnostic, Indianapolis, IN, USA). After exposure, the film was developed using a Konica SRX-101A developing machine.

\section{RESULTS}

\section{Strategy of Site Specific Integration, Design of Insertion Site and TALENs}

Transgene expression varies greatly due to position effects of random insertion in transgenic plants. Targeted insertion of a transgene into a specific and active location of the chromosome is desirable for both line selection and downstream characterization. Our approach used TAL effector nucleases to introduce targeted DSB in the potato genome immediately following an endogenous promoter, so that the provided cassette harboring a native promoter-less herbicide resistant marker and transgene of interest can be captured in that site. The target site chosen for this study was the intron region within the $5^{\prime}$ UTR of the potato Ubi7 gene. As shown in Figure 1, once inserted into the desired site, the endogenous Ubi7 promoter will drive the expression of the promoter-less marker gene which will then confer herbicide resistance and enable the regeneration of transgenic plants on medium containing the appropriate selective agent. For this study, the marker gene used was the mutated acetolactate synthase gene (ALS) (also known as acetohydroxy acid synthase, or AHAS). ALS catalyzes the first step in the synthesis of the branched-chain amino acids and is sensitive to a number of herbicides such as imazamox. Amino acid substitutions provide tolerance to the herbicides in a number of species including potato (Andersson et al., 2003). We engineered two amino acid substitutions in the potato StALS gene, designated $m S t A L S$. The DNA and protein sequences with nucleotide and amino acid substitutions are shown in Supplementary Figures S1 and S2.

We chose the potato Ubi7 gene as our target site because it is constitutively expressed in potato plants and has a promoter that is commonly used for constitutive gene expression in potato transgenic research and applications (Richael et al., 2008). Ubi7 transcripts have an intron in the $5^{\prime}$ UTR. The intron along with the first monomer have been shown to contribute to high expression levels of the transgene (Garbarino et al., 1995). Therefore to promote high expression of our transgene, we included the intron and first monomer in the design of the promoter-less herbicide resistant gene. A pair of TAL effector nucleases were designed to make a DSB within the intron, which was (a) $>25$-bp upstream from the branch site [consensus $=\mathrm{CU}(\mathrm{A} / \mathrm{G}) \mathrm{A}(\mathrm{C} / \mathrm{U})]$, pyrimidine-rich ( = AT-rich) region, and intron/exon junction (consensus = CAGG), and (b) > 50-bp downstream from the splice donor site at the exon/intron junction (consensus = AGGT) (Lou et al., 1993). The sequence of the target region is shown in Supplementary Figure S3. The TALEN scaffold used in our experiments is plant codon optimized Hax3 (Mahfouz et al., 2011), a member of the AvrBs3 family that was identified in Brassicaceae pathogen Xanthamonas campestris pv. armoraciae strain 5 (Kay et al., 2005). Other modifications made to the scaffold included: (a) truncation of the C-terminal activation domain of the original Hax3 (last 215 amino acids); (b) addition of a NLS from SV40 virus at the N terminal of the truncated Hax3 protein (Lassner et al., 1991). The DNA and protein sequences of the final forward and reverse TALENs can be found in the supplementary data (Supplementary Figures S4-S7).

A binary vector was created for transient expression of the TALENs in plant cells. This vector contained two TALENs each operably linked to a strong constitutive promoter [Cauliflower Mosaic Virus (CaMV) 35s or Figwort Mosaic Virus] and followed by a terminator [nopaline synthase (Nos) or octopine synthase] to form two separate plant expression cassettes. The two cassettes were cloned into a pSIM binary vector to form pSIM2170 as shown in Figure 2. To facilitate transient expression of the effectors, the left border (LB) was removed from pSIM2170. A plant hormone cytokinin biosynthesis isopentenyl transferase (ipt) gene expression cassette was included at the left side of the TALEN cassettes. If stable transformation occurs, the entire vector sequence is integrated into the plant cell's genome. The stable integration of the ipt expression cassette results in the overproduction of cytokinin in cells and shoots. These shoots are stunted and fail to develop roots (Richael et al., 2008), which allows for stably integrated TALEN sequences to be eliminated based on phenotype. This reduces the number of plants with stably integrated effector expression cassettes into the plant genome for further analysis.

\section{Validation of the Designed TALEN in Transient Expression System}

To demonstrate the efficiency of the designed TALENs and their ability to recognize the target sequence and induce a DSB, we took advantage of a transient infiltration method in $N$. benthamiana. For this purpose, a target vector pSIM2167 was designed for co-infiltration into $N$. benthamiana with the TALEN construct previously described (pSIM2170). The target vector contains a modified $\beta$-glucuronidase (GUS) reporter gene driven by a constitutive FMV promoter. The GUS ATG start codon immediately precedes a 60 bp target sequence containing both forward and reverse recognitions sites. Between the two recognition sequences, there is a stop codon in frame with the GUS coding sequence which rendered this form of the GUS gene non-functional (Figure 3, Top panel). Binary vectors pSIM2170 and pSIM2167 were transformed into Agrobacterium strain AGL1 and subsequently co-infiltrated or 


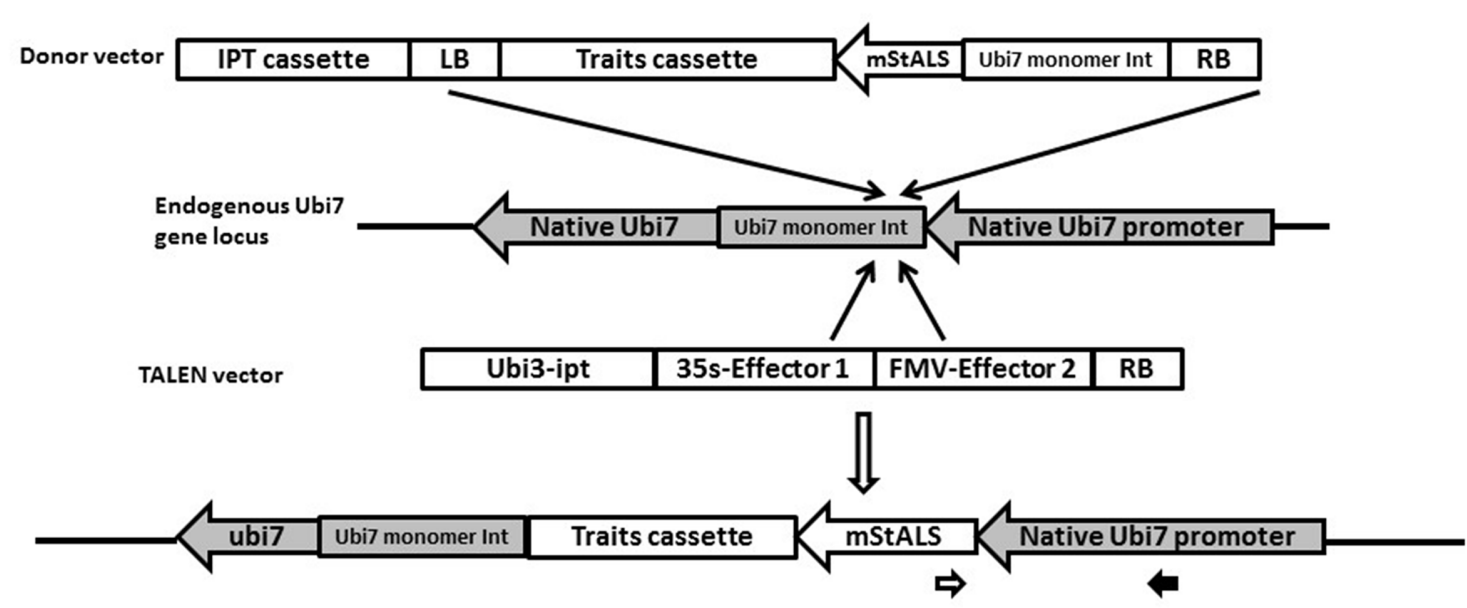

FIGURE 1 | Schematic of strategy for the targeted T-DNA insertion. The TALEN vector contains a pair of TALENs targeting the endogenous potato Ubi7 intron immediately $5^{\prime}$ to the initiation codon for the first ubiquitin monomer. The LB was removed and an ipt overexpression cassette was put next to the TALEN expression cassettes on the left side of T-DNA. The donor vector contains a promoter-less mutated potato ALS gene (mStALS) next to the RB and the nptll cassette. Upon Agrobacterium-mediated transformation, expression of TALENs generate DSB at the endogenous target, once the donor T-DNA is inserted into this endogenous site, the endogenous promoter will drive the expression of mStALS expression and resulted targeted events can be selected out on herbicide containing medium. The targeted events can also be PCR confirmed by using a primer in the mStALS gene (opened arrow) paired with a primer within the endogenous Ubi7 promoter (solid arrow).

\section{\begin{tabular}{|l|l|l|l|l|l|l|l|}
\hline Ubi3P & IPT & Ubi3T & 355 \\
\hline
\end{tabular}}

FIGURE 2 | Schematic of binary vector pSIM2170 that contains forward and reverse TALENs. Two TALENs were driven by either 35 s or FMV constitutive promoter followed by either OCS or Nos terminator, respectively. Each TALEN has a SV40 nuclear localization sequence (NLS), designed repeat variable diresidue (RVD) (E3 or E4 repeat), and truncated C-terminal fused to a Fokl cleavage domain. A potato Ubi3 promoter-driven ipt gene cassette was put on the left side of T-DNA and the T-DNA LB was removed in this vector.

infiltrated individually into $N$. benthamiana leaves. Infiltrated leaf disks were collected 2-3 days post infiltration and GUS staining was performed. Leaves infiltrated with pSIM2167 alone showed no GUS expression, demonstrating that the stop codon present in the spacer region led to the inactivation of GUS due to early termination of the coding sequence (Figure 3, Middle left panel). When leaves were co-infiltrated with the TALEN construct pSIM2170 and the target construct pSIM2167, the TALENs induced DSBs and cleaved the region containing the stop codon in the target sequence and were subsequently repaired by $N$. benthamiana cellular DNA repair NHEJ machinery. This in many cases resulted in the loss of the stop codon. When the repaired form of the target sequence was in frame with the downstream GUS coding sequence, GUS protein function was recovered and visualized by a standard GUS staining assay (Jefferson et al., 1987) (Figure 3, Middle right panel). GUS expression results indicated that the designed TALENs cleaved the stop codon in the target region and was successfully repaired by the cellular repair mechanism.

To further validate the functionality of the designed TAL effectors, sequence analysis was performed on leaves 3 days post infiltration. The target sequence region was PCR amplified and sequenced to identify TALEN mediated mutations. To accommodate for the unpredictable nature of the transient assay with the newly designed TALENs, a four nucleotide restriction enzyme site $(A l u I)$ was included between the two recognition sites. Direct PCR and cloning of the target sequence yielded large amounts of unmodified target sequence. The genomic DNA isolated from the infiltrated tissue was therefore digested with AluI enzyme prior to PCR amplification to reduce the quantity of unmodified target sequence. The PCR product underwent a second round of digestion with AluI enzyme to further enrich the modified target sequence prior to cloning and sequence analysis. Target site sequence analysis showed no modifications present in the tissue infiltrated with pSIM2167 alone (data not shown). However, individual clones obtained from the samples co-infiltrated with pSIM2170 and pSIM2167 shown in Figure 3 Bottom panel confirmed TALEN mediated modifications in the target region. Of the 50 clones sequenced, 4 had unmodified target sequences, one contained a single base pair substitution, one contained a single base pair insertion, and 44 harbored deletions ranging from 2 to $15 \mathrm{bp}$ (Figure 3, Bottom panel). 

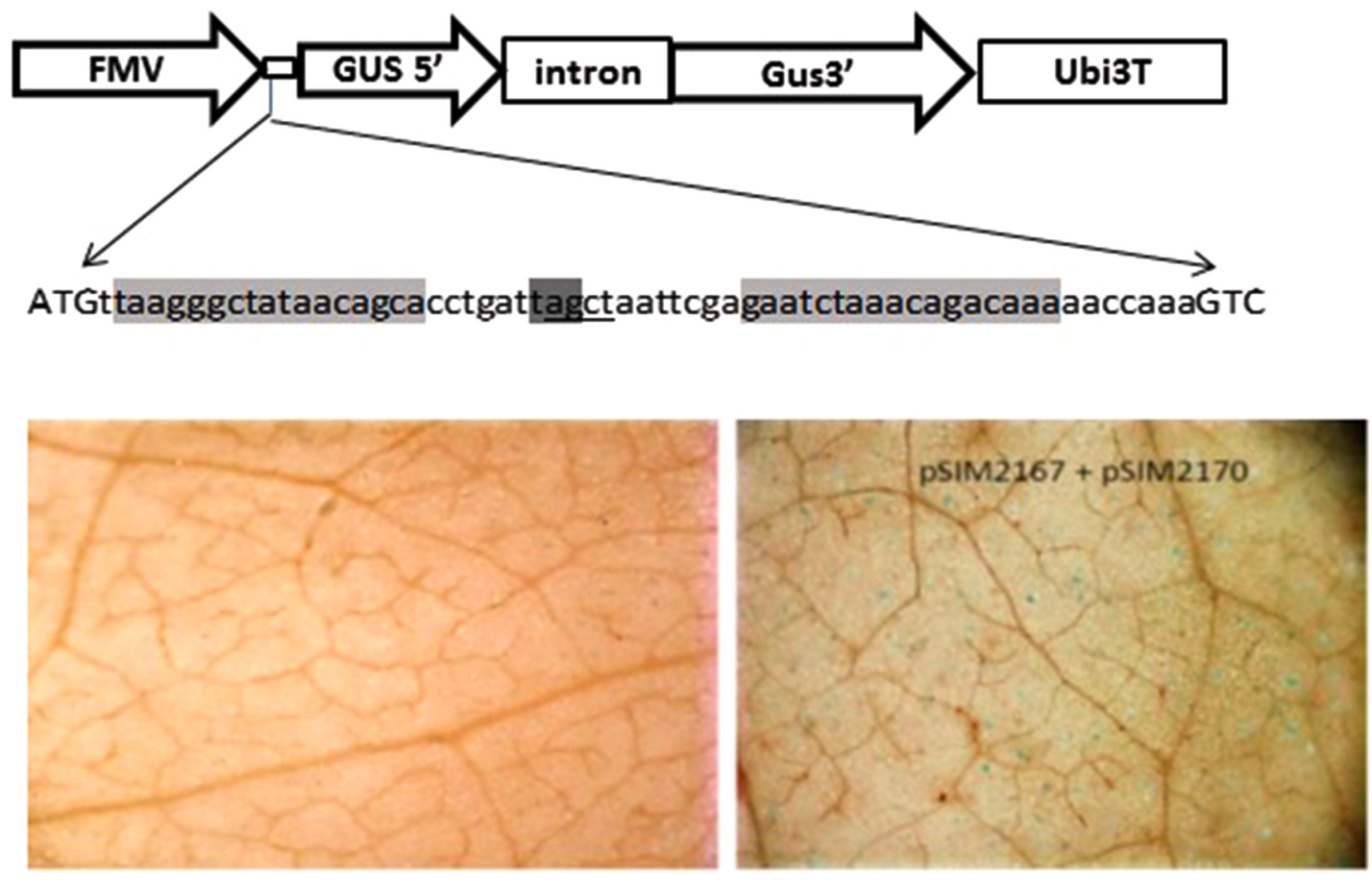

Target
$599(4)$
$116(1)$
$118(1)$
$112(3)$
$602(5)$
$604(4)$
106
$588(4)$
$598(5)$
$121(4)$
$108(5)$
$590(6)$
$593(2)$

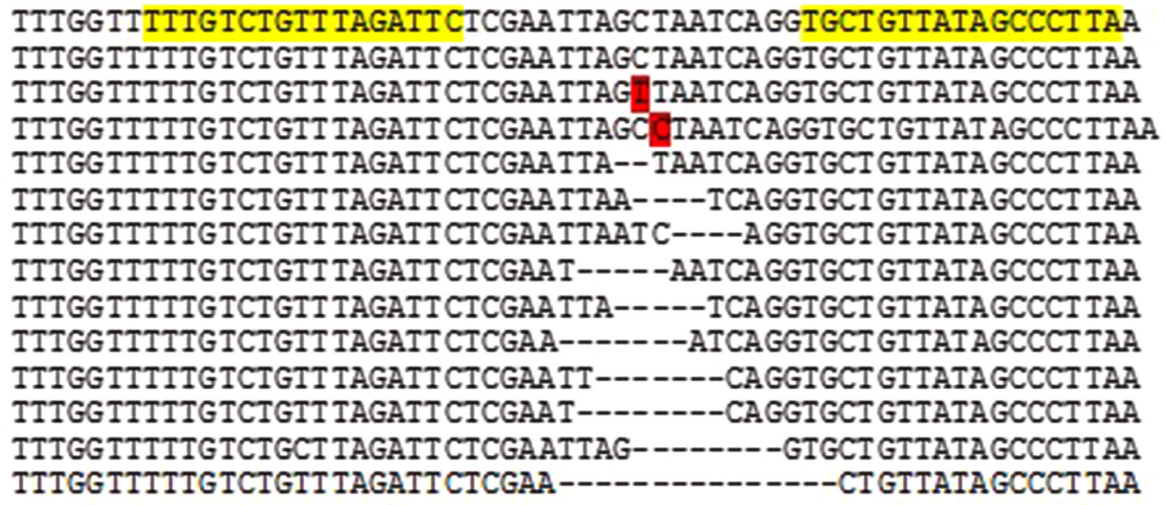

FIGURE 3 | Verification of designed TALENs in $\boldsymbol{N}$. benthamiana infiltration. (Top panel) Target vector pSIM2167 of TALENs for N. benthamiana infiltration. A 60 bp DNA sequence from StUbi7 5' intron was inserted between the first and second amino acid codons of the GUS gene. There is an in frame stop codon (dark gray highlighted) in the spacer region between the two recognition sites (light gray highlighted) which will abolish the GUS protein translation. TALEN-mediated DNA cleavage and subsequent NHEJ based repairing will destroy the stop codon and translation of the GUS will be in frame and restore GUS activity. An Alul restriction enzyme site (underlined) was also included in the spacer to facilitate detection of TALEN-mediated indels. (Middle and bottom panels): The functionality of designed TALEN in transient assay. Target construct was Agro-infiltrated into N. benthamiana leaves alone or together with the TALEN construct. Forty-eight hours after infiltration, leaf tissue at the infiltrated site was collected and stained with GUS staining solution and de-stained with ethanol for microscopic examination. DNA was isolated from co-infiltrated tissue, digested with Alul, PCR amplified, cloned and sequenced. (Middle left panel): Target construct pSIM2167 alone. (Middle right panel): Target construct pSIM2167 co-infiltrated with TALEN construct pSIM2170. (Bottom panel): Sequences of target region with various modifications. The number in the parenthesis represents how many times the modification occurred in the 50 clones sequenced. The numbers on the left are identifiers of the respective clones.

\section{Integration of Gene of Interest Cassette into Targeted Location in Potato Genome}

After functional confirmation of the designed TALENs in the $N$. benthamiana transient system, we tested the ability of the designed TALENs to facilitate targeted transgene integration of the provided insert cassette into the potato genome. We designed a donor vector, pSIM2370 comprised of two cassettes: the first cassette contains a promoter-less herbicide resistant gene, mutated potato acetolactate synthase ( $m S t A L S)$ gene, and the second consists of a selection marker (nptII) expression 


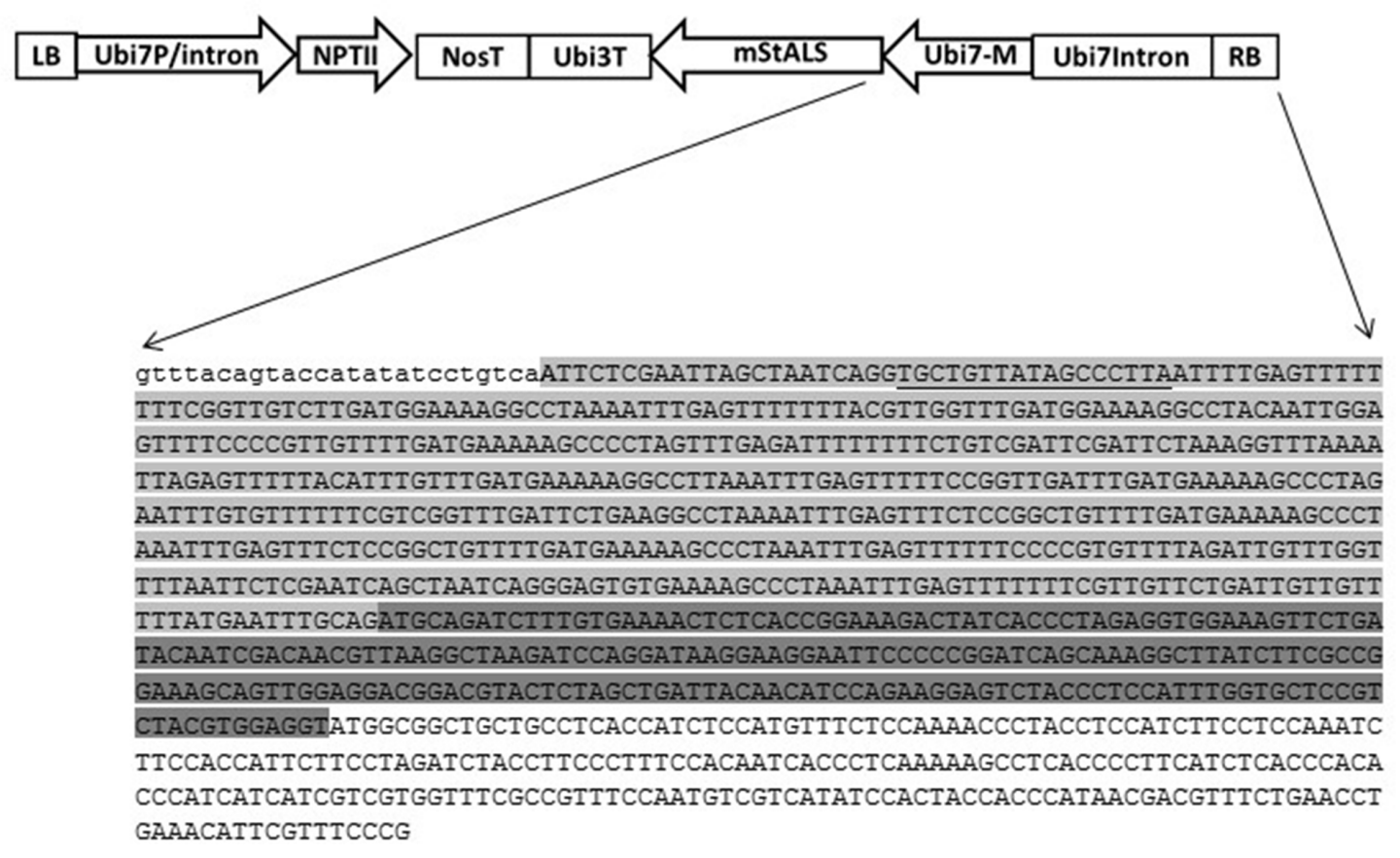

FIGURE 4 | Design of knock-in donor vector. The donor vector pSIM2370 T-DNA contains a StUbi7 promoter driven npt/l gene cassette at the LB side, and a promoter-less mStALS gene at the RB side. At the sequence level, next to the RB, are sequences of a partial Ubi7 5' intron region (light gray highlighted), Ubi7 monomer (dark gray highlighted) fused to the mStALS coding sequence (only partial sequence is shown in this figure). The intron sequence contains a spacer between the two TALEN binding sites and the second TALEN recognition site (underlined). There is no ipt expression cassette on the backbone of donor vector.

cassette (Figure 4). Following the right border, the sequence comprised of: part of the StUbi7 gene $5^{\prime}$-UTR intron, a spacer sequence located between the two targeted TAL binding sites, a Ubi7 monomer-encoding sequence fused to mStALS gene that is insensitive to ALS inhibitor and a terminator of the potato ubiquitin-3 gene. The $5^{\prime}$ sequence of this cassette is shown in Figure 5. The second plant antibiotic resistant gene (nptII) expression cassette included a nos terminator, an nptII coding region and a Ubi7 promoter near the LB. A total of 900 Russet Ranger potato tissue culture explants were cotransformed with the TAL effector nuclease vector pSIM2170 and donor vector pSIM2370 as described in the experimental procedures. When the provided transgene was integrated into the targeted genome position, regenerated transgenic plants contained the promoter-less herbicide resistant gene driven by the endogenous Ubi7 promoter. In our experiment, we generated a total of 63 independent herbicide resistant lines for further characterization.

\section{Characterization of Herbicide Resistant Transgenic Lines}

To further validate the integration of the T-DNA into the targeted location, the herbicide resistant plants were characterized through PCR sequence analysis. Primers were designed to span the integration region, with the forward primer located in the endogenous Ubi7 promoter and the reverse in the $\mathrm{mStALS}$ transgene. Integration of the target gene generates a $1.1 \mathrm{~kb}$ product. Complications during integration such as
DNA re-arrangements, extra fragment insertions or deletions changes the size of the product and were visualized on an agarose gel. As shown in Figure 6 middle panel, among the first 28 herbicide resistant lines screened by targeted insertion specific primers, 27 had a PCR product at the expected size (96\%). We cloned and sequenced 19 PCR products with the expected sizes as well as the larger PCR product from line 25. We found that the transgene integrated into the endogenous Ubi7 intron region without any additional nucleotides in all 19 lines (Supplementary Figure S8). Line No. 25 produced a larger PCR product and when sequenced was found to contain an insert of an additional segment of the Ubi7 intron (Supplementary Figure S8).

Targeted integration in herbicide resistant plants was further confirmed by Southern blot analysis. The assay was performed using HindIII digested genomic DNA and probed with a StALS coding sequence. The HindIII site is located in the promoter region of Ubi7 gene and in the ALS gene coding region (Figure 5, top panel). DNA from transgenic lines digested with HindIII and probed with the partial StALS sequence upstream of the HindIII site should produce a targeted event specific band $(2 \mathrm{~kb})$ on a Southern blot. As shown in Figure 5 bottom panel, four bands were generated in the wild type control. These bands are signals from the endogenous StALS genes. There are additional bands in all PCR positive herbicide resistant lines at the expected $2 \mathrm{~kb}$ size for targeted insertion. Although every event had a targeted insertion, many also contained multiple copies as indicated by additional bands on the Southern blot. To select lines for further 

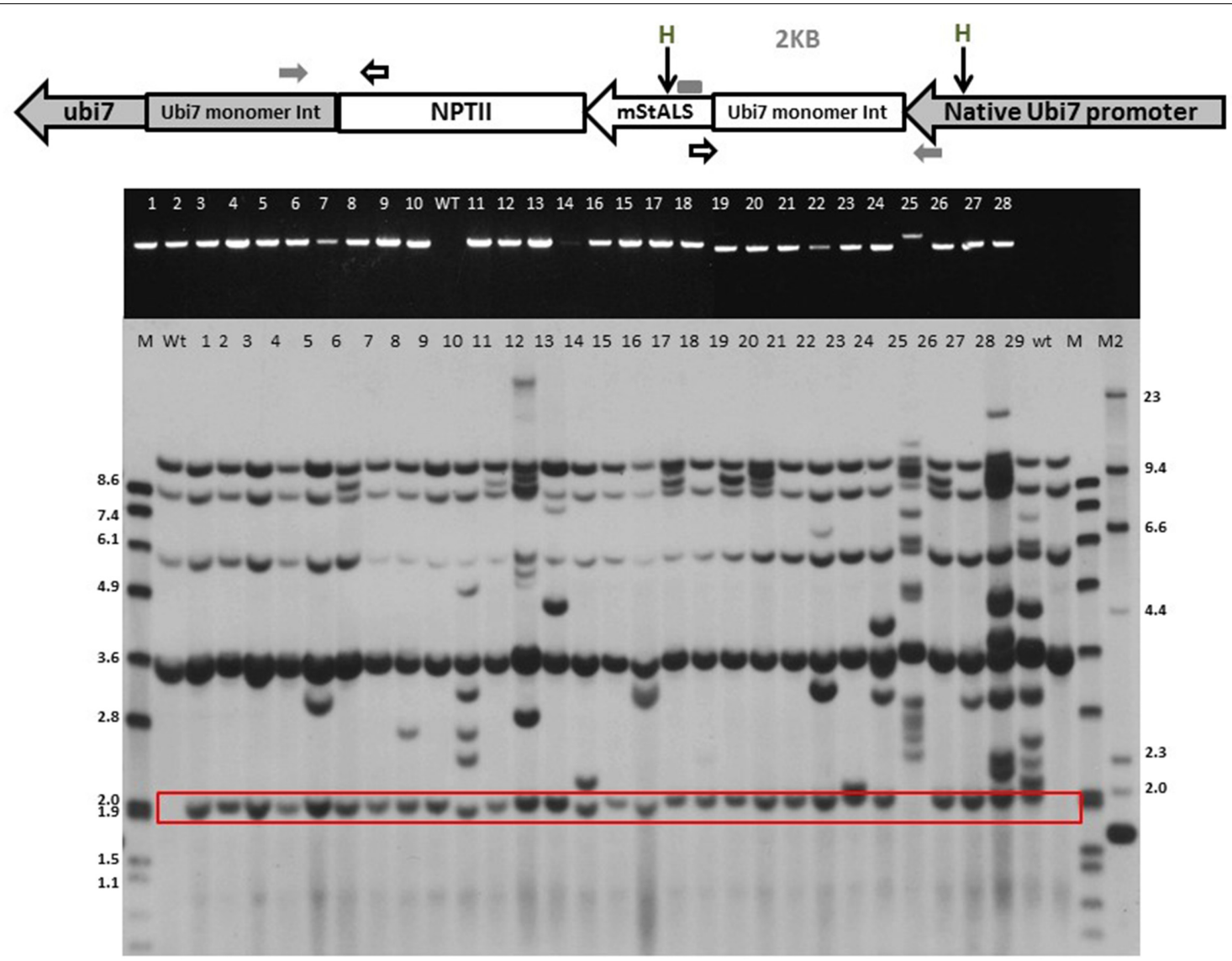

FIGURE 5 | Identification of targeted integration events. DNA from herbicide resistant transgenic lines were isolated for PCR and Southern analyses. (Top panel) Schematic of the Ubi7 locus after site-specific gene integration. Primers 1: within the endogenous Ubi7 promoter and 2: within the $m S t A L S$ gene coding region are indicated by arrows. Two Hindlll restriction enzyme sites, one in the endogenous Ubi7 promoter and one in the mStALS coding region are also labeled. (Middle panel): PCR using primers 1 and 2 detects site-specific integration events. (Bottom panel): Southern blot using StALS probe detects the site-specific integration band at predicted size (boxed). The size of dig-labeled ladders were marked as kb (M: Dig-labeled marker 7, M2: Dig-labeled marker 2).

investigation, the data from the Southern blots were used to determine single copy lines as opposed to PCR products. Among the 12 selected single copy lines, an additional Southern blot was performed with a probe that targeted the TALEN expression cassette. We found that only one line contained a small piece of the expression cassette. This result validated that the negative selection method utilizing the ipt gene eliminated the majority of transgenic lines containing TALEN construct (Supplementary Figure S9).

Additionally, we investigated the integration at the LB junction. If the T-DNA was inserted into the designed genome location, the T-DNA LB flanking sequence would be the endogenous Ubi7 gene intron and monomer. We performed PCR flanking the LB sequence in all twelve single copy lines with a primer pair designed to amplify this region. We were able to amplify a product from 5 lines. As shown in Figure 6, all products aligned to a deduced junction sequence with variations at the LB cutting site. Three lines appeared to have a predicted cut at the LB cleavage site (between the 3rd and 4th nucleotides from $3^{\prime}$ end of border sequence, Van Haaren et al., 1988) and was linked to the intron of the endogenous $U b i 7$ gene with three base pair filler nucleotides. Two sequences had a small deletion at the LB, the first fused directly to endogenous Ubi7 intron without filler DNA and the second fused to the endogenous Ubi7 intron with short filler DNA.

\section{DISCUSSION}

In this study, we demonstrated the ability to use TALEN induced DSB to integrate a GOI cassette into a specific target site in the potato genome. To facilitate the selection of integrated events, we chose a well characterized endogenous potato $\mathrm{Ubi} 7$ promoter to drive the expression of a promoter-less marker gene.

In theory, any designed transgene with a promoter-less marker gene could be inserted immediately following a constitutive promoter to allow expression of the marker gene and other gene(s) of interest within the same T-DNA. To select for targeted insertion, we used the plant-derived mStALS gene as the selection marker. By choosing a native marker, we are able to integrate genes in a cisgenic manner since our entire cassette could originate from sexually compatible species; e.g., late blight 


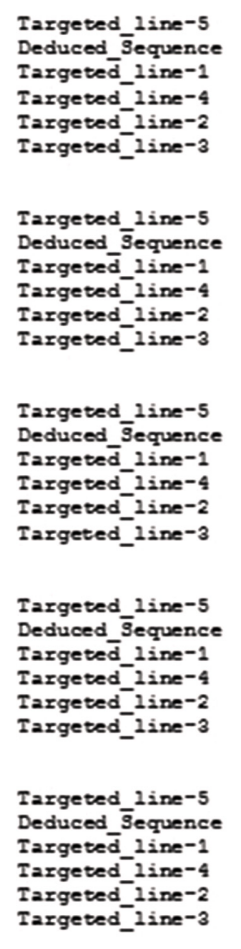

ITCGCTCATGIGITGACATATAAGAAACCTIAGLATGIATTTGIATITGTAAAMACT 60 ITCGCTCATGIGTIGAGCATATAAGAAACCTIAGLATGTAITTGIATTIGTAAAATACT 60

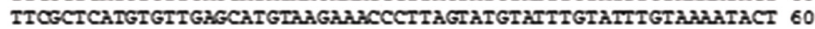
TTCGCTCATGTGTTGACATATAAGAACCCTTAGIATGTAITTGTATTTGTAAAMACT 60 TTCGCTCATGTGTTGAGCATATAAGAAACCTTAGIATGTAITTGTATTTGTAAAATACT 60 ITCGCTCATGIGTTGAGCATATAAGAACCCTIAGATGIATTIGIATITGLAAATACT 60 TICGCTCATGIGITGAGCATATAAGAAACCCTIAGATGIATTTGIATITGIAAAMACT

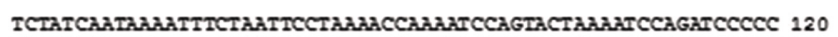

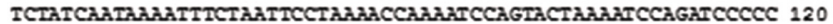
TCLATCAATAhAATTTCTAATTCCTAAAAChAhATCCAGTACThAAATCCAGATCCCCC 120 ICTRC) TCTATCAATAAATTTCTAATTCCTAAACCAAAATCCAGTACTAAAATCCAGATCCCCC 120

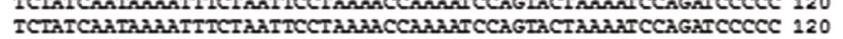
1

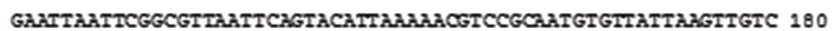

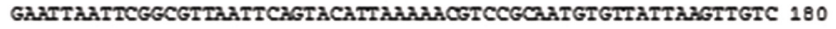
GAATIAATTCGGCGTIAATTCAGIACATIAAAAACGTCCGGAIGIGITATTAAGTTGTC 180

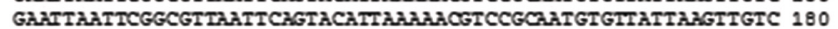

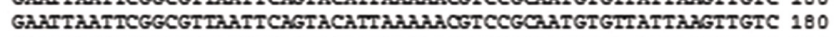

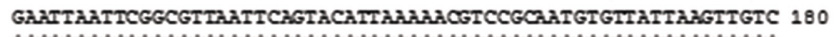

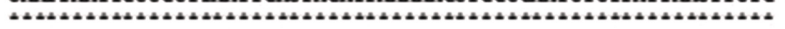

TAAGCGTCAITIGTITACACG-CAATATATCCTGTCA-------ATICTCGATTAGC 232 TAAGCGICAATITGITIACACOA-CAATATATCCTGCCA-G-----AITCTCGATIAGC 233 TAAGCGTCAATTIGTTIACACCA-CAATATATCCTGICA------ATICTCGATTAGC 232 TAAGCGTCAATTGITIACACG-CAATATATCCTGTCA--------ATTCTCGATTAGC 232 -----AaTrCTCGATTAGC 195

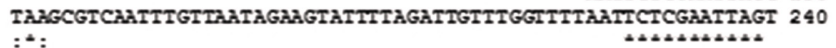

TAMCAGGTGCTGITAIAGCCCTTAATIITGAGTITTCTTCGGTTGTCTTGATGGAAAג 292 TAATCAGGTGCTGITATAGCCCTAAITITGAGITITITTTCGITGITITGATGGAAAA 293 TAMTCAGGIGCTGITAIAGCCCITAATITIGAGITITITTICGGTGICTIGATGGAAAA 292

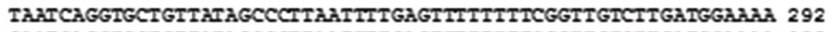

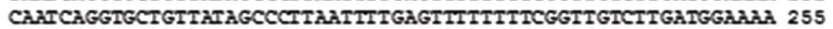
TAATCAGGTGCTGITATAGCCCTIAATITTGAGITITITTCGGTTGTCTIGATGGAAAA 300

FIGURE 6 | Sequence of LB side flanking region. A pair of primers, one within the endogenous Ubi7 intron and the other within the T-DNA region of the donor vector close to the left border (as shown in Figure 5), were used to amplify targeted insertion in the LB junctions. Amplified products were cloned and sequenced. Sequences of five lines are shown here and aligned to deduce the LB junction after targeted integration. The LB sequence is green highlighted in the deduced sequence with the last three nucleotides red-highlighted, which are lost in the perfect LB cleavage events.

genes from wild potato species. Other native markers such as plant derived 5-enolpyruvylshikimate-3-phosphate synthase (epsps) gene can also be used as a selection marker or used as a marker for re-transformation of an additional gene(s) (Sidorov and Duncan, 2009). Although it is possible to achieve site specific integration and selection, non-specific integration can still occur. To circumvent this issue, integration specific PCR and selection of single copy lines were used to screen out the events with unintended non-specific insertions.

When gene editing tools are used to generate a DSB, gene replacement/insertion is generally thought to occur via the $\mathrm{HR}$ pathway, which requires flanking homologous arms for insertion. Salomon and Puchta (1998) have previously shown that DSBs serve as a target site for T-DNA integration when the breaks are generated by the restriction enzyme I-SceI. It has also been reported that T-DNA can be captured and integrated into a DSB site via NHEJ pathway (Chilton and Que, 2003 and Tzfira et al., 2003). We therefore hypothesized that when a DSB is generated at a desired genomic location, there will be an increased likelihood that the T-DNA will integrate into that site, thus reducing the need for lengthy homologous arms. Shortening the length of homologous arms decreases the complexity of the donor construct. In our design, we fused the Ubi7 monomer and part of the $5^{\prime}$-UTR intron to the marker gene. This gave our insert partial homology to the endogenous target site at the right border (RB). The majority of the homologous sequences are after the designed cutting site which differs from the typical homologous arm design. Our results indicated the possibility of achieving site specific gene insertion to a target site without the use of long homologous arms. We also observed fusion of the endogenous Ubi7 sequence to our insert without additional nucleotides. This suggested that the integration of the insert at the RB site occurred through HR (Supplementary Figure S8). When we investigated the LB junction, we observed imperfect integration between the LB sequence of our construct and the endogenous Ubi7 gene. The LB junction was either perfectly cut or contained filler nucleotides or small deletions. This occurrence is similar to that observed in regular T-DNA insertion junctions (De Buck et al., 1999). This suggests that our insertion events occurred via one-sided (RB) HR. The LB was fused to endogenous sequence downstream of the DSB in a NHEJ manner. Similarly, Puchta et al. (1996) demonstrated in planta, HR restricted to one side of the DSB could happen which was described as the non-conservative onesided invasion model. However, for the identified single copy lines, not every LB junction was identified by PCR using our current primers. This was most likely due to the occurrence of large deletions resulting from NHEJ that caused the loss of primer binding sites. To bypass this issue, primers to amplify a larger region could be used. There could also be unknown mechanisms involved in LB integration making it difficult to detect the junction. Nevertheless, our results showed that in several single copy targeted events, it is feasible to identify the LB 
junction region using simple PCR methods (Five representative lines in Figure 6). Together with the observation that we obtained many lines that have multiple copies of random T-DNA insertion suggest that there are opportunities to improve the design of our donor vector. Previous studies conducting precise targeting events in maize and cotton did not observe additional random integrations (D'Halluin et al., 2008, 2013). However, this was not the case in our experiments which could possibly be attributed to the use of a different plant species. Our next steps will focus on donor architectures that can reduce the non-specific random T-DNA integration and increase the ratio of precise integration at the LB side.

Current research typically utilizes genome editing enzymes through stable integration in the plant. The gene that encodes the genome editing enzyme can be subsequently segregated out in the following generation. However, when working with a vegetatively propagated species such as potato, segregating the next generation is not feasible. In this study, we used a negative selection based approach to ensure transient expression of the genome editing nucleases during the transformation process. To do this, we designed the T-DNA region of nucleases plasmid to contain a plant hormone cytokinin biosynthesis cassette $(i p t)$ alongside the TALEN cassettes. Ideally, when the TALEN cassette integrates into the plant genome, the ipt cassette will also be integrated. This will result in a phenotypically distinguishable plant that can be screened visually and discarded. Continual modification of the genome can occur with the presence of stably integrated TALENs, however by eliminating lines with stable integration of TALEN, they are unable to continue modifying the genome. This suggests that the lines containing the modifications underwent alterations during the regeneration process, most likely before first cell division occurred. This method could also be adapted to other genome editing tools such as ZFN, meganucleases and CRISPR/Cas9 system. Especially for CRISP/Cas9, given the simplicity of the system, knock-in gene of interest(s) into different genomic location is simpler since the designing of new nucleases is not needed. In our lab, when CRISPR/Cas9 are designed to cleave the same target described in this paper, similar T-DNA integration results were achieved (unpublished data).

We hypothesized that among the independent lines, if targeted transgene insertion is achieved, the variability of transgene expression levels should be minimal. For this purpose, 12 single copy targeted events were selected for an expression study. Northern blot results showed that expression of the nptII gene, the trait gene in our POC donor vector, in targeted lines was relatively uniform (Supplementary Figure S10). This is consistent to other targeted studies that utilized site-specific recombinase such as Cre-lox, FLP-FRT and ZFN (Srivastava et al., 2004; Nandy and Srivastava, 2011; Kumar et al., 2015). Currently, we have designed new donor vectors which contains a trait gene of interest and trait genes in both targeted and random events will be compared. However, the targeted insertion events could generate some uncharacteristic phenotypes. This could be due to somaclonal variation that often occurs during the cell dedifferentiation process. It has been shown in the Cre-lox site specific recombinase system that identical sitespecific transgene integration is able to produce alleles that are differentially silenced. This is attributed to factors like epigenetic modifications such as methylation (Day et al., 2000). For that reason, we do not expect all targeted events to have the same phenotype. On the other hand, provided a good integration site is chosen, fewer events will be required if targeted integration is achieved.

Since a functional Ubi7 promoter is used in the donor vector to drive the nptII gene expression, we also tested the possibility that this promoter activates the promoter-less $m S t A L S$ gene. The donor vector (pSIM2370) was transformed into potato plants alone using kanamycin as selection agent. All 50 kanamycin resistant lines obtained were also grown on imazamox containing medium, and only three lines (none were single copy) survived. The ratio of resistant lines falls within the range of a promoter trapping experiment in which the T-DNA is trapped by an endogenous promoter. This indicated that the Ubi7 promoter used to drive $n p t I I$ gene expression did not activate the promoterless $m S t A L S$ gene in the same T-DNA.

In summary, we report a method that relies on the transient expression of TALEN to generate transgenic lines with sitespecifically integrated T-DNA. Additionally, single copy events with easily determined flanking sequences and consistent transgene expression can aid in the ease of characterization. As a result, this could support transgenic crop improvement with more precision, thereby greatly reducing the number of independent lines to be generated and the workload of downstream characterization.

\section{AUTHOR CONTRIBUTIONS}

HD designed the work, $\mathrm{HD}$ and AF conducted the experiments, analyzed and interpreted data, TW performed plant transformation. HD and AF drafted manuscript, and HD, AF, $\mathrm{CR}$, and TW reviewed, edited and approved manuscript.

\section{ACKNOWLEDGMENTS}

The authors are thankful to Dr. Caius Rommens for his support on the initiation of the project. Ms. Kristi Severson for plant transformation, Ms. Michele Krucker, Ms. Rebecca Kirkpatrick for growing N. benthamiana plants and Mr. Nic Nottingham for molecular support on random insertion lines. A corresponding patent application on the subject matter has been filed with U.S. Serial Number 14/084,406.

\section{SUPPLEMENTARY MATERIAL}

The Supplementary Material for this article can be found online at: http://journal.frontiersin.org/article/10.3389/fpls.2016.01572 


\section{REFERENCES}

Ainley, W. M., Sastry-Dent, L., Welter, M. E., Murray, M. G., Zeitler, B., Amora, R., et al. (2013). Trait stacking via targeted genome editing. Plant Biotechnol. J. 11, 1126-1134. doi: 10.1111/pbi.12107

Albert, H., Dale, E. C., Lee, E., and Ow, D. W. (1995). Site-specific integration of DNA into wild-type and mutant lox sites placed in the plant genome. Plant J. 7 , 649-659. doi: 10.1046/j.1365-313X.1995.7040649.x

Andersson, M., Trifonova, A., Andersson, A. B., Johansson, M., Bülow, L., and Hofvander, P. (2003). A novel selection system for potato transformation using a mutated AHAS gene. Plant Cell Rep. 22, 261-267. doi: 10.1007/s00299-0030684-8

Bogdanove, A. J., and Voytas, D. F. (2011). TAL effectors: customizable proteins for DNA targeting. Science 333, 1843-1846. doi: 10.1126/science.1204094

Bortesi, L., and Fischer, R. (2015). The CRISPR/Cas9 system for plant genome editing and beyond. Biotechnol. Adv. 33, 41-52. doi: 10.1016/j.biotechadv.2014.12.006

Cai, C. Q., Doyon, Y., Ainley, W. M., Miller, J. C., Dekelver, R. C., Moehle, E. A., et al. (2009). Targeted transgene integration in plant cells using designed zinc finger nucleases. Plant Mol. Biol. 69, 699-709. doi: 10.1007/s11103-008-9449-7

Chilton, M. D., and Que, Q. (2003). Targeted integration of T-DNA into the tobacco genome at double-stranded breaks: new insights on the mechanism of T-DNA integration. Plant Physiol. 133, 956-965. doi: 10.1104/pp.103.026104

Day, C. D., Lee, E., Kobayashi, J., Holappa, L. D., Albert, H., and Ow, D. W. (2000). Transgene integration into the same chromosome location can produce alleles that express at a predictable level, or alleles that are differentially silenced. Genes Dev. 15, 2869-2880. doi: 10.1101/gad.849600

De Buck, S., Jacobs, A., Van Montagu, M., and Depicker, A. (1999). The DNA sequences of T-DNA junctions suggest that complex T-DNA loci are formed by a recombination process resembling T-DNA integration. Plant J. 20, 295-304. doi: 10.1046/j.1365-313X.1999.t01-1-00602.x

De Paepe, A., De Buck, S., Nolf, J., Van Lerberge, E., and Depicker, A. (2013). Sitespecific T-DNA integration in Arabidopsis thaliana mediated by the combined action of CRE recombinase and $\phi \mathrm{C} 31$ integrase. Plant J. 75, 172-184. doi: 10.1111/tpj.12202

De Pater, S., Pinas, J. E., Hooykaas, P. J., and van der Zaal, B. J. (2013). ZFNmediated gene targeting of the Arabidopsis protoporphyrinogen oxidase gene through Agrobacterium-mediated floral dip transformation. Plant Biotechnol. J. 11, 510-515. doi: $10.1111 /$ pbi. 12040

D’Halluin, K., Vanderstraeten, C., Stals, E., Cornelissen, M., and Ruiter, R. (2008). Homologous recombination: a basis for targeted genome optimization in crop species such as maize. Plant Biotechnol. J. 6, 93-102.

D'Halluin, K., Vanderstraeten, C., Van Hulle, J., Rosolowska, J., Van Den Brande, I., Pennewaert, A., et al. (2013). Targeted molecular trait stacking in cotton through targeted double-strand break induction. Plant Biotechnol. J. 11, 933941. doi: $10.1111 /$ pbi. 12085

Epinat, J. C., Arnould, S., Chames, P., Rochaix, P., Desfontaines, D., Puzin, C., et al. (2003). A novel engineered meganuclease induces homologous recombination in yeast and mammalian cells. Nucleic Acids Res. 31, 2952-2962. doi: 10.1093/nar/gkg375

Fukushige, S., and Sauer, B. (1992). Genomic targeting with a positive-selection lox integration vector allows highly reproducible gene expression in mammalian cells. Proc. Natl. Acad. Sci. U.S.A. 89, 7905-7909. doi: 10.1073/pnas.89.17.7905

Gamborg, O. L. (1966). Aromatic metabolism in plants II. Enzymes of the skikimate pathway in suspension cultures of plant cells. Can. J. Biochem. 44, 791-799.

Garbarino, J. E., Oosumi, T., and Belknap, W. R. (1995). Isolation of a polyubiquitin promoter and its expression in transgenic potato plants. Plant Physiol. 109, 1371-1378. doi: 10.1104/pp.109.4.1371

Golic, M. M., Rong, Y. S., Petersen, R. B., Lindquist, S. L., and Golic, K. G. (1997). FLP-mediated DNA mobilization to specific target sites in Drosophila chromosomes. Nucleic Acids Res. 25, 3665-3671. doi: 10.1093/nar/25.18.3665

Hanin, M., and Paszkowski, J. (2003). Plant genome modification by homologous recombination. Curr. Opin. Plant Biol. 6, 157-162. doi: 10.1016/S13695266(03)00016-5

Jefferson, R. A., Kavanagh, T. A., and Bevan, M. W. (1987). GUS fusion: $\beta$-glucuronidase as a sensitive and versatile gene fusion marker in higher plants. EMBO J. 6, 3901-3907.
Jinek, M., Chylinski, K., Fonfara, I., Hauer, M., Doudna, J. A., and Charpentier, E. (2012). A programmable dual-RNA-guided DNA endonuclease in adaptive bacterial immunity. Science 337, 816-821. doi: 10.1126/science.1225829

Kay, S., Boch, J., and Bonas, U. (2005). Characterization of AvrBs3-like effectors from a Brassicaceae pathogen reveals virulence and avirulence activities and a protein with a novel repeat architecture. Mol. Plant Microbe Interact. 18, 838-848. doi: 10.1094/MPMI-18-0838

Kempin, S. A., Liljegren, S. J., Block, L. M., Rounsley, S. D., Yanofsky, M. F., and Lam, E. (1997). Targeted disruption in Arabidopsis. Nature 389, 802-803. doi: $10.1038 / 39770$

Kim, Y. G., Shi, Y., Berg, J. M., and Chandrasegaran, S. (1997). Site-specific cleavage of DNA-RNA hybrids by zinc finger/FokI cleavage domain fusions. Gene 203, 43-49. doi: 10.1016/S0378-1119(97)00489-7

Kumar, S., AlAbed, D., Worden, A., Novak, S., Wu, H., Ausmus, C., et al. (2015). A modular gene targeting system for sequential transgene stacking in plants. J. Biotechnol. 207, 12-20. doi: 10.1016/j.jbiotec.2015.04.006

Lassner, M. W., Jones, A., Daubert, S., and Comai, L. (1991). Targeting of T7 RNA polymerase to tobacco nuclei mediated by an SV40 nuclear location signal. Plant Mol. Biol. 17, 229-234. doi: 10.1007/BF00039497

Li, H. Q., Terada, R., Li, M. R., and Iida, S. (2004). RecQ helicase enhances homologous recombination in plants. FEBS Lett. 574, 151-155. doi: 10.1016/j.febslet.2004.08.020

Li, J. F., Norville, J. E., Aach, J., McCormack, M., Zhang, D., Bush, J., et al. (2013). Multiplex and homologous recombination-mediated genome editing in Arabidopsis and Nicotiana benthamiana using guide RNA and Cas9. Nat. Biotechnol. 31, 688-691. doi: 10.1038/nbt.2654

Li, T., Huang, S., Jiang, W. Z., Wright, D., Spalding, M. H., Weeks, D. P., et al. (2011). TAL nucleases (TALNs): hybrid proteins composed of TAL effectors and FokI DNA-cleavage domain. Nucleic Acids Res. 39, 359-372. doi: $10.1093 /$ nar/gkq704

Lida, S., and Terada, R. (2005). Modification of endogenous natural genes by gene targeting in rice and other higher plants. Plant Mol. Biol. 59, 205-219. doi: 10.1007/s11103-005-2162-x

Lou, H., McCullough, A. J., and Schuler, M. A. (1993). 3’ splice site selection in dicot plant nuclei is position dependent. Mol. Cell. Biol. 13, 4485-4493. doi: 10.1128/MCB.13.8.4485

Mahfouz, M. M., Li, L., Shamimuzzaman, M., Wibowo, A., Fang, X., and Zhu, J. K. (2011). De novo-engineered transcription activator-like effector (TALE) hybrid nuclease with novel DNA binding specificity creates double-strand breaks. Proc. Natl. Acad. Sci. U.S.A. 108, 2623-2628. doi: 10.1073/pnas.1019533108

Murashige, T., and Skoog, F. (1962). A revised medium for rapid growth and bioassays with tobacco tissue cultures. Physiol. Plant. 15, 431-497. doi: 10.1111/j.1399-3054.1962.tb08052.x

Mussolino, C., and Cathomen, T. (2013). RNA guides genome engineering. Nat. Biotechnol. 31, 208-209. doi: 10.1038/nbt.2527

Nandy, S., and Srivastava, V. (2011). Site-specific gene integration in rice genome mediated by the FLP-FRT recombination system. Plant Biotechnol. J. 9, 713721. doi: 10.1111/j.1467-7652.2010.00577.x

Ow, D. W. (2011). Recombinase-mediated gene stacking as a transformation operating system. J. Integr. Plant Biol. 53, 512-519. doi: 10.1111/j.17447909.2011.01061.x

Peach, C., and Velten, J. (1991). Transgene expression variability (position effect) of CAT and GUS reporter genes driven by linked divergent T-DNA promoters. Plant Mol. Biol. 17, 49-60. doi: 10.1007/BF00036805

Porebski, S., Bailey, L. G., and Baum, B. R. (1997). Modification of a CTAB DNA extraction protocol for plants containing high polysaccharide and polyphenol components. Plant Mol. Biol. Rep. 15, 8-15. doi: 10.1007/BF027 72108

Porteus, M. H., and Baltimore, D. (2003). Chimeric nucleases stimulate gene targeting in human cells. Science 300:763. doi: 10.1126/science.1078395

Puchta, H., Dujon, B., and Hohn, B. (1993). Homologous recombination in plant cells is enhanced by in vivo induction of double strand breaks into DNA by a site-specific endonuclease. Nucleic Acids Res. 21, 5034-5040. doi: $10.1093 / \mathrm{nar} / 21.22 .5034$

Puchta, H., Dujon, B., and Hohn, B. (1996). Two different but related mechanisms are used in plants for the repair of genomic double-strand breaks by homologous recombination. Proc. Natl. Acad. Sci. U.S.A. 93, 5055-5060. doi: 10.1073/pnas.93.10.5055 
Reiss, B., Schubert, I., Köpchen, K., Wendeler, E., Schell, J., and Puchta, H. (2000). RecA stimulates sister chromatid exchange and the fidelity of double-strand break repair, but not gene targeting, in plants transformed by Agrobacterium. Proc. Natl. Acad. Sci. U.S.A. 97, 3358-3363. doi: 10.1073/pnas.97.7.3358

Richael, C. M., Kalyaeva, M., Chretien, R. C., Yan, H., Adimulam, S., Stivison, A., et al. (2008). Cytokinin vectors mediate marker-free and backbone-free plant transformation. Transgenic Res. 17, 905-917. doi: 10.1007/s11248-008-9175-6

Salomon, S., and Puchta, H. (1998). Capture of genomic and T-DNA sequences during double-strand break repair in somatic plant cells. EMBO J. 17, 60866095. doi: 10.1093/emboj/17.20.6086

Shalev, G., Sitrit, Y., Avivi-Ragolski, N., Lichtenstein, C., and Levy, A. A. (1999). Stimulation of homologous recombination in plants by expression of the bacterial resolvase ruvC. Proc. Natl. Acad. Sci. U.S.A. 96, 7398-7402. doi: 10.1073/pnas.96.13.7398

Shan, Q., Wang, Y., Li, J., Zhang, Y., Chen, K., Liang, Z., et al. (2013). Targeted genome modification of crop plants using a CRISPR-Cas system. Nat. Biotechnol. 31, 686-688. doi: 10.1038/nbt.2650

Shukla, V. K., Doyon, Y., Miller, J. C., DeKelver, R. C., Moehle, E. A., Worden, S. E., et al. (2009). Precise genome modification in the crop species Zea mays using zinc-finger nucleases. Nature 459, 437-441. doi: 10.1038/nature07992

Sidorov, V., and Duncan, D. (2009). Agrobacterium-mediated maize transformation: immature embryos versus callus. Methods Mol. Biol. 526, 47-58. doi: 10.1007/978-1-59745-494-0_4

Srivastava, V., Ariza-Nieto, M., and Wilson, A. J. (2004). Cre-mediated site-specific gene integration for consistent transgene expression in rice. Plant Biotechnol. J. 2, 169-179. doi: 10.1111/j.1467-7652.2003.00061.x

Svitashev, S., Young, J. K., Schwartz, C., Gao, H., Falco, S. C., and Cigan, A. M. (2015). Targeted mutagenesis, precise gene editing, and site-specific gene insertion in maize using cas9 and guide RNA. Plant Physiol. 169, 931-945. doi: 10.1104/pp.15.00793

Symington, L. S., and Gautier, J. (2011). Double-strand break end resection and repair pathway choice. Annu. Rev. Genet. 45, 247-271. doi: 10.1146/annurevgenet-110410-132435

Terada, R., Johzuka-Hisatomi, Y., Saitoh, M., Asao, H., and Iida, S. (2007). Gene targeting by homologous recombination as a biotechnological tool for rice functional genomics. Plant Physiol. 144, 846-856. doi: 10.1104/pp.107.095992
Terada, R., Urawa, H., Inagaki, Y., Tsugane, K., and Iida, S. (2002). Efficient gene targeting by homologous recombination in rice. Nat. Biotechnol. 20, 1030-1034. doi: $10.1038 /$ nbt737

Townsend, J. A., Wright, D. A., Winfrey, R. J., Fu, F., Maeder, M. L., Joung, J. K., et al. (2009). High-frequency modification of plant genes using engineered zinc-finger nucleases. Nature 459, 442-445. doi: 10.1038/nature07845

Tzfira, T., Frankman, L. R., Vaidya, M., and Citovsky, V. (2003). Sitespecific integration of Agrobacterium tumefaciens T-DNA via double-stranded intermediates. Plant Physiol. 133, 1011-1023. doi: 10.1104/pp.103.032128

Van Haaren, M. J. J., Sedee, N. J. A., Krul, M., Schilperoort, R. A., and Hooykaas, P. J. J. (1988). Function of heterologous and pseudo border repeats in T region transfer via the octopine virulence system of Agrobacterium tumefaciens. Plant Mol. Biol. 11, 773-781. doi: 10.1007/BF00019517

Wang, X., Viret, J. F., Eldridge, A., Perera, R., Signer, E. R., and Chiurazzi, M. (2001). Positive-negative selection for homologous recombination in Arabidopsis. Gene 272, 249-255. doi: 10.1016/S0378-1119(01)00532-7

Wright, D. A., Townsend, J. A., Winfrey, R. J. Jr., Irwin, P. A., Rajagopal, J., Lonosky, P. M., et al. (2005). High-frequency homologous recombination in plants mediated by zinc-finger nucleases. Plant J. 44, 693-705. doi: 10.1111/j.1365-313X.2005.02551.x

Xie, K., and Yang, Y. (2013). RNA-guided genome editing in plants using a CRISPR-Cas system. Mol Plant. 6, 1975-1983. doi: 10.1093/mp/sst119

Zhang, Y., Zhang, F., Li, X., Baller, J. A., Qi, Y., Starker, C. G., et al. (2013). Transcription activator-like effector nucleases enable efficient plant genome engineering. Plant Physiol. 161, 20-27. doi: 10.1104/pp.112.205179

Conflict of Interest Statement: The authors declare that the research was conducted in the absence of any commercial or financial relationships that could be construed as a potential conflict of interest.

Copyright (c) 2016 Forsyth, Weeks, Richael and Duan. This is an open-access article distributed under the terms of the Creative Commons Attribution License (CC BY). The use, distribution or reproduction in other forums is permitted, provided the original author(s) or licensor are credited and that the original publication in this journal is cited, in accordance with accepted academic practice. No use, distribution or reproduction is permitted which does not comply with these terms. 